\title{
Sterile Neutrino Search with the Double Chooz Experiment
}

\section{Tsunayuki Matsubara*}

Tokyo Metropolitan University, Japan

E-mail: matsubara@hepmail.phys.se.tmu.ac.jp

\section{Denise Hellwig}

RWTH Aachen University, Germany

E-mail: hellwig@physik.rwth-aachen.de

Double Chooz is a reactor antineutrino disappearance experiment located in Chooz, France. A far detector at a distance of about $1 \mathrm{~km}$ from reactor cores is operating since 2011; a near detector of identical design at a distance of about $400 \mathrm{~m}$ is operating since begin 2015. Beyond the precise measurement of $\theta_{13}$, Double Chooz has a strong sensitivity to so called light sterile neutrinos. Sterile neutrinos are neutrino mass states not taking part in weak interactions, but may mix with known neutrino states. In this paper, we present an analysis method to search for sterile neutrinos and the expected sensitivity with the baselines of our detectors.

38th International Conference on High Energy Physics 3-10 August 2016

Chicago, USA

\footnotetext{
* Speaker.
} 


\section{Experimental Setup}

Double Chooz is a reactor neutrino experiment located in Chooz France designed to measure the neutrino mixing angle $\theta_{13}$ [1]. It consists of two identical liquid scintillator detectors. The near detector (ND) at about $400 \mathrm{~m}$ distance from the two Chooz B reactor cores is operating since begin 2015. The far detector (FD) at about $1050 \mathrm{~m}$ distance is operating since April 2011. Electron antineutrinos from the reactors are identified via the inverse beta decay (IBD) process prompt delayed signal on Gadolinium (Gd) capture. This setup is used to search for additional hypothetical neutrino states, so called light sterile neutrinos, motivated by anomalies observed by e.g. LSND [2] and MiniBooNE [3] and the reactor neutrino anomaly [4].

\section{Sterile Neutrino Oscillations}

The so called $3+1$ model assumes one additional sterile, i.e. not weakly interacting, neutrino state. This introduces three new neutrino mixing angles $\theta_{14}, \theta_{24}$, and $\theta_{34}$, along with one new mass. Relevant for the electron (anti) neutrino survival probability $P_{\bar{v}_{e} \rightarrow \bar{v}_{e}}$ are only $\theta_{14}$ and $\Delta m_{41}^{2}$ [5]. $P_{\bar{v}_{e} \rightarrow \bar{v}_{e}}$ is approximately given by

$$
P_{\bar{v}_{e} \rightarrow \bar{v}_{e}} \approx 1-\cos ^{4} \theta_{14} \sin ^{2} 2 \theta_{13} \sin ^{2}\left(\frac{1.267 L \Delta m_{31}^{2}}{E_{v}}\right)-\sin ^{2} 2 \theta_{14} \sin ^{2}\left(\frac{1.267 L \Delta m_{41}^{2}}{E_{v}}\right),
$$

where $E_{V}$ is the neutrino energy and $L$ the baseline. Note that in this analysis the full four flavor oscillation probabilities obtained with GLoBES [6][7] and its add-on for sterile neutrinos and nonstandard interactions [8][9] are used.

Figure 1 shows as an example the neutrino IBD-candidate prediction for $\sin ^{2} 2 \theta_{14}=0.1$ and $\Delta m_{41}^{2}=0.1 \mathrm{eV}^{2}$ for FD (left) and ND (right) relative to no-oscillation. Statistics corresponds to three years physics runtime resulting in about 240000 events in the ND and about 40000 events in the FD during the double detector period which is reffered to as FD2. The FD dataset of the single detector period, i.e. before 2015, is reffered to as FD1 and contains about 20000 events.
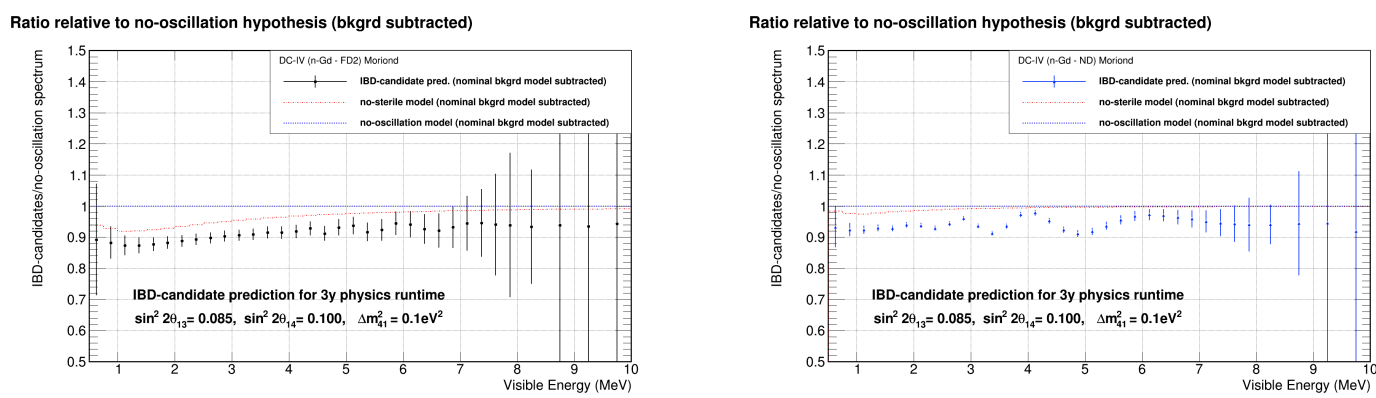

Figure 1: IBD-candidate predictions relative to no-oscillation for an example sterile neutrino scenario for FD (left) and ND (right), where the predictions are (nominal) background subtracted i.e. it is the neutrino prediction only. Statistics corresponds to three years physics runtime. The no-sterile case is shown in red. 


\section{Analysis Method}

The sterile analysis is done via a Poisson Likelihood forward folding approach using 40 prompt energy bins. It uses the ND, FD2 and FD1 dataset. In addition, there is a reactor-off sample for FD1.

Two different approaches are used. In the MC-Data approach reactor flux shape and reactor flux normalization are constrained by the Monte-Carlo (MC). In contrast, for the Data-Data approach the global reactor flux normalization is free in each energy bin. Other systematics similar for both approaches.

Backgrounds are handled as nuisance parameters where FD backgrounds are additionally constrained with the FD reactor-off sample. The detection systematics uncertainty is accounted for by one nussiance parameter per dataset. Energy scale conversion from visible prompt energy $E_{v i s, p r o m p t}$ in the detector to initial neutrino energy $E_{v}$ is done using $E_{v i s, p r o m p t}=a+b E_{v}+c E_{v}^{2}$. Investigated parameters are $\theta_{14}$ and $\Delta m_{41}^{2}$ where $\theta_{14}$ and $\theta_{13}$ are fitted simultaneously. $\Delta m_{32}^{2} \approx \Delta m_{31}^{2}$ is constraint with a Gaussian prior.

\section{Expected Sensitivity for Three Years Operation}

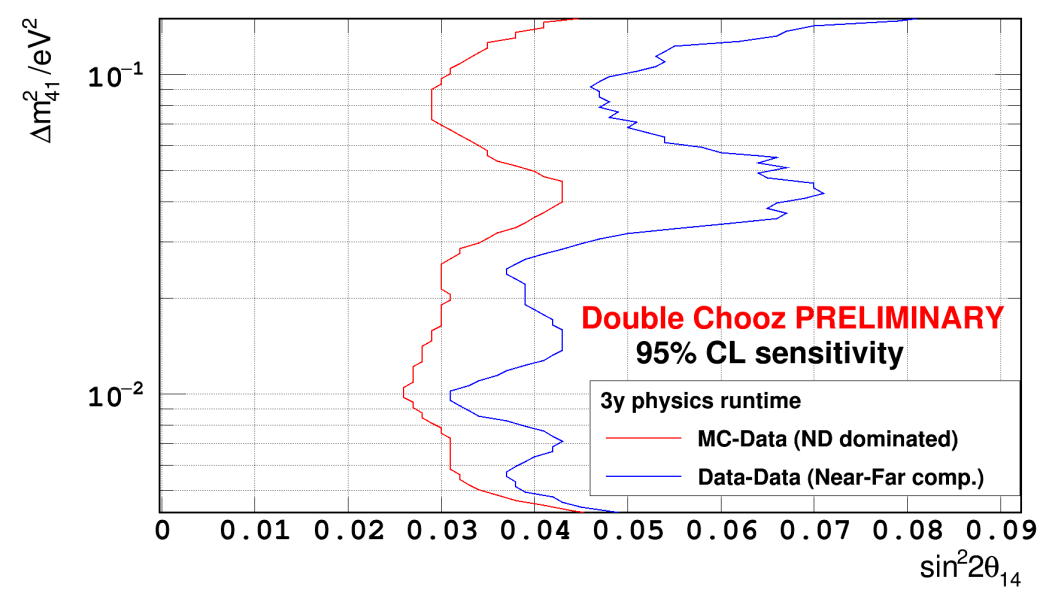

Figure 2: Expected sensitivity for three years physics data taking with both detectors. The MC-Data approach is shown in red. It compares IBD-candidate rate and shape of both detectors to the MC prediction. The Data-Data approach shown in blue does not use the global reactor flux normalization and shape from MC. Instead, it only compares the IBD-candidate rate and shape of both detectors to each other; comparision to $\mathrm{MC}$ is not done.

Figure 2 shows the expected sensitivity for three years physics data taking with both detectors. The MC-Data approach is shown in red while the Data-Data approach is shown in blue. As already metioned in section 3, the MC-Data approach compares IBD candidate rate normalization and shape of both detectors to the MC prediction, where the uncertainty on reactor flux is $2.34 \%$. In contrast, the Data-Data approach only compares the IBD candidate rate and shape of both detectors. Importantly, the global reactor flux normalization and shape from MC is not used. 
The most important difference between MC-Data and Data-Data approach is the performance with respect to the disagreement between data and prediction in the 4-6 $\mathrm{MeV}$ region reported by Daya Bay, Double Chooz and Reno which is referred to as spectral distortion [1][10][11].

Importantly, in the Data-Data approach the spectral distortion chancels out while in the MCData approach it does not chancel out. Therefore, the Data-Data approach is not biased by the spectral distortion but the MC-Data approach may only be applied to data if and only if the spectral distortion is understood.

The largest differnence in sensitivity of both approaches is observed for large $\Delta m_{41}^{2}$. The DataData is running out of sensitivity for large $\Delta m_{41}^{2}$ as oscillation effects are smearing out more and more with increasing $\Delta m_{41}^{2}$ until only a smaller reactor flux normalization is observed with both detectors. In contrast, as the MC-Data approach compares the global reactor flux normalization to MC, the MC-Data approach has a larger sensitivity for large $\Delta m_{41}^{2}$.

The valley in both sensitivities at around $\Delta m_{41}^{2} \approx 10^{-1.4} \mathrm{eV}^{2}$ is caused by interference of ND baselines. It is more prominent in the Data-Data approach, because this approach cannot use the global deficit resulting from canceling out oscillation patterns while the MC-Data approach can.

\section{Summary and Outlook}

The Double Chooz experiment has the capability to search for light sterile neutrinos using the electron antineutrino channel. We reported expected the sensitivity with the Data-Data and the MC-Data approach using IBD-candidates on Gadolinium capture.

In addition to the IBD-candidates on Gadolinium capture, IBD-candidates on Hydrogen $(\mathrm{H})$ capture may be used. This would increase the statistics and therefore the sensitivity. Splitting up data into subsamples depending on which off the two reactors cores were running would reduce interference effects between different baselines and would therefore improve the sensitivity.

\section{References}

[1] Y. Abe, et al., JHEP 10, 086 (2014). [Erratum: JHEP02,074(2015)].

[2] A. Aguilar-Arevalo, et al., Phys. Rev. D64, 112007 (2001).

[3] A. A. Aguilar-Arevalo, et al., Phys. Rev. Lett. 110, 161801 (2013).

[4] G. Mention, et al., Phys. Rev. D83, 073006 (2011).

[5] J. Kopp, P. A. N. Machado, M. Maltoni, T. Schwetz, JHEP 05, 050 (2013).

[6] P. Huber, M. Lindner, W. Winter, Comput. Phys. Commun. 167, 195 (2005).

[7] P. Huber, J. Kopp, M. Lindner, M. Rolinec, W. Winter, Comput. Phys. Commun. 177, 432 (2007).

[8] J. Kopp, Int. J. Mod. Phys. C19, 523 (2008). Erratum ibid. C19 (2008) 845.

[9] J. Kopp, M. Lindner, T. Ota, J. Sato, Phys. Rev. D77, 013007 (2008).

[10] F. P. An, et al., Phys. Rev. Lett. 116, 061801 (2016).

[11] J. H. Choi, et al., Phys. Rev. Lett. 116, 211801 (2016). 